\title{
Diagnóstico de parásitos en heces del huemul (Hippocamelus bisulcus) en el centro y sur de Chile
}

\author{
Ana Hinojosa-Sáez ${ }^{凶(\mathbb{0})}$ M.Sc; Marco Pérez $\mathrm{S}^{2} \otimes \mathbb{C}$ MV; Rodrigo López $\mathrm{R}^{3} \otimes(\mathbb{0}$ Tec. Pesquero;

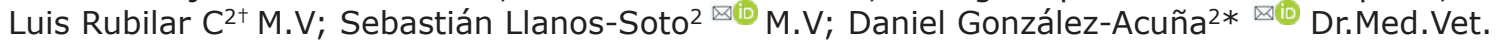

${ }^{1}$ Corporación Nacional Forestal, Departamento de Patrimonio Silvestre, Claudio Arrau 738, Chillán, Chile.

¿Universidad de Concepción, Facultad de Ciencias Veterinarias, Vicente Méndez 595, Chillán, Chile.

${ }^{3}$ Comité Nacional pro Defensa de la Flora y Fauna, Anibal Pinto 215, Concepción, Chile.

*Correspondencia: danigonz@udec.cl

Recibido: Septiembre 2018; Aceptado: Febrero 2019; Publicado: Junio 2019.

\section{RESUMEN}

Objetivo. Identificar parásitos en heces del huemul (Hippocamelus bisulcus) presente en áreas naturales protegidas del centro y sur de Chile y evaluar diferencias en la prevalencia de parásitos entre regiones y estaciones del año. Materiales y métodos. 200 muestras de heces de huemul fueron colectadas de distintas localidades de las regiones de Ñuble, Aysén y Magallanes a lo largo de todas las estaciones entre los años 2006 y 2008. Las muestras fueron analizadas utilizando las técnicas de flotación y sedimentación, para ser posteriormente observadas en el microscopio con el objetivo de detectar parásitos. Adicionalmente, se analizaron 124 de estas muestras para determinar la presencia de Fasciola hepatica. Resultados. El 38.0\% $(n=76 / 200)$ de las muestras analizadas fueron positivas a algún parásito, observándose una prevalencia de $11.8 \%(n=2 / 17)$ en Nuble, $22.8 \%(n=28 / 123)$ en Aysén y un $76.7 \%(n=46 / 60)$ en Magallanes. En Ñuble se encontraron huevos de Moniezia sp., mientras que en Aysén y Magallanes se identificaron huevos tipo Estrongílido, Nematodirus sp. y ooquistes de Eimeria spp. No se evidenció F. hepatica en las muestras analizadas. Se detectan diferencias de parasitismo entre las estaciones $(p \leq 0.05)$ en Aysén y Magallanes. Conclusiones. La diferencia en la prevalencia y diversidad parasitaria entre regiones incluidas en este estudio pueden estar relacionadas con la disimilitud en la densidad poblacional de huemules y la masa ganadera de cada región. Es necesario continuar con monitoreos parasitológicos en poblaciones de huemul en Chile sur y central y estudiar la relación de esta especie con el ganado y la posible transmisión inter- e intraespecífica de parásitos.

Palabras clave: Amenazado, Cervidae, conservación, parasitología, vida silvestre (Fuente: ICYT, SKOS).

\section{ABSTRACT}

Objective. Identify parasites in huemul (Hippocamelus bisulcus) feces from natural protected areas of south-central Chile and evaluate differences in parasite prevalence between regions and seasons. Materials and methods. 200 fecal samples from huemul were collected during all seasons of the year from different localities of the Ñuble, Aysén and Magallanes regions between years 2006 and 2008. Samples were analyzed using flotation and sedimentation techniques and further observed with the microscope in order to detect parasites. Additionally, 124 samples were analyzed to determine the presence of Fasciola hepatica. Results. The $38.0 \%(n=76 / 200)$ of samples were positive to at least one parasite form, evidencing a prevalence of $11.8 \%(n=2 / 17)$ in Ñuble, $22.8 \%(n=28 / 123)$ in Aysén and a $76.7 \%(n=46 / 60)$ in Magallanes. Moniezia sp. eggs were found in the Ñuble region, meanwhile, Strongyle-type eggs, Nematodirus sp. eggs and Eimeria spp. oocysts were found in the Aysén and Magallanes regions. There was no evidence of $F$. hepatica during sample examination. Differences in parasitism were detected between seasons in Aysén and Magallanes $(p \leq 0.05)$. Conclusions. Aysén and Magallanes regions showed a relatively higher prevalence and diversity of parasites than the Ñuble region. This could be related to differences in density of huemul populations and livestock in each region. Further parasitological surveys in huemul populations from central and southern Chile should be performed in the future. Studies involving the relationship between the huemul and livestock and the potential inter- and intraspecific transmission of parasites are also required.

Keywords: Cervidae, conservation, endangered, parasitology, wildlife (Source: ICYT, SKOS).

Como citar (Vancouver)

Hinojosa-Sáez A, Pérez SM, López RR, Rubilar CL, Llanos-Soto S, González-Acuña D. Diagnóstico de parásitos en heces del huemul (Hippocamelus bisulcus) en el centro y sur de Chile. Rev MVZ Cordoba. 2019; 24(2):7268-7272. DOI: https://doi.org/10.21897/rmvz.1389

(C)EI (los) autor (es), Revista MVZ Córdoba 2019. Este artículo se distribuye bajo los términos de la licencia internacional Creative Commons Attribution 4.0 (https://creativecommons.org/licenses/by-sa/4.0/), que permite el uso sin restricciones, la distribución y la reproducción en cualquier medio, siempre que se otorgue el crédito apropiado al autor o autores originales y la fuente. 


\section{INTRODUCCIÓN}

El huemul (Hippocamelus bisulcus) es una especie de ciervo amenazado presente en áreas andino-patagónicas predominantes en sotobosques poco densos en vegetación de Chile y Argentina (1). Las poblaciones de huemul son pequeñas y se encuentran dispersas a lo largo de su rango de distribución en Chile, estando presentes en las regiones de Ñuble, Los Lagos, Los Ríos, Aysén y Magallanes $(1,2)$. Actualmente, el huemul enfrenta problemas relacionados con la actividad antrópica, como la fragmentación y pérdida de hábitat e introducción de enfermedades exóticas (2). El huemul en Chile central es considerado en riesgo crítico de extinción debido a la reducción continua de su población, con estimaciones que indican la existencia de menos de 50 individuos para la región de Ñuble, lo que puede resultar en consecuencias relacionadas con una variabilidad genética reducida, como la endogamia y la deriva genética $(1,2)$. En contraste, las poblaciones de huemul al sur de Chile se caracterizan por poseer densidades que van desde 2,2 a 6,9 individuos $/ \mathrm{km}^{2}$ en áreas aisladas (1). En esta zona, las condiciones climáticas y topográficas han limitado la presencia humana y actividad agrícola, lo que ha permitido a los huemules mantener poblaciones más numerosas y densas que sus conespecíficos presentes en áreas naturales de Chile central (1).

La presencia de ganado en áreas protegidas y no protegidas de Chile central y sur es una amenaza continua a las poblaciones de huemul, debido a que los animales de granja compiten por recursos alimenticios, modifican el paisaje y son una fuente importante de enfermedades infecciosas y parásitos (2). Entre los parásitos reportados para el huemul se encuentran Moniezia sp., tipo Estrongílido, Nematodirus sp., Dictyocaulus sp., Trichuris sp., Eimeria spp., Taenia hydatigena, Fasciola hepatica y Ostertagia sp. $(3,4,5)$.

El objetivo principal de este estudio fue identificar parásitos presentes en las heces del huemul procedentes de diferentes áreas naturales protegidas de Chile sur y central. Adicionalmente, se evaluaron las diferencias en prevalencia de parásitos en poblaciones de huemul de diferentes regiones y en distintas estaciones del año.

\section{MATERIALES Y MÉTODOS}

Recolección de muestras. Un total de 200 muestras de heces frescas de huemul fueron recolectadas entre los años 2006 y 2008 desde reservas (RN) y parques nacionales (PN) localizados en tres regiones de Chile 17 en Ñuble, 123 en Aysén y 60 en Magallanes (Figura 1). Las muestras fueron almacenadas individualmente en bolsas de plástico hermético y transportadas en cajas termales, las cuales fueron mantenidas a $4^{\circ} \mathrm{C}$ en el Laboratorio de Parásitos y Enfermedades de Fauna Silvestre, Facultad de Ciencias Veterinarias, Universidad de Concepción, Chillán. El tiempo desde la colecta hasta el análisis fue como máximo 5 días.

Las muestras fueron obtenidas durante todas las estaciones del año: 72 en otoño ( 8 en Ñuble, 21 en Aysén y 43 en Magallanes), 51 en invierno (3 en Ñuble, 42 en Aysén y 6 en Magallanes), 62 en primavera ( 3 en Ñuble, 55 en Aysén y 4 en Magallanes) y 15 en verano ( 3 en Ñuble, 5 en Aysén y 7 en Magallanes).
Las muestras de la región de Ñuble fueron obtenidas en áreas comprendidas en la zona andina de esta región, específicamente de la RN Ñuble $\left(37^{\circ} 00^{\prime} 45^{\prime \prime} S\right.$, $\left.71^{\circ} 28^{\prime} 51^{\prime \prime O}\right)$, río Ñuble (36⒊'12"S, $\left.71^{\circ} 23^{\prime} 24^{\prime \prime} \mathrm{O}\right)$ y RN Los Huemules de Niblinto (36 $\left.46^{\prime} 32^{\prime \prime} S, 71^{\circ} 27^{\prime} 04^{\prime \prime} \mathrm{O}\right)$. Este distrito posee un clima frío templado con bosques caducifolios de Nothofagus. En Aysén, las muestras fueron obtenidas en las cercanías del río Nef $\left(47^{\circ} 06^{\prime} 55^{\prime \prime} \mathrm{S}\right.$, $\left.72^{\circ} 55^{\prime} 32^{\prime \prime} \mathrm{W}\right)$, Tortel $\left(47^{\circ} 48>32 » \mathrm{~S}, 7^{\circ} 29>13 \gg 0\right)$, Villa $\mathrm{O}^{\prime}$ Higgins $\left(48^{\circ} 27>140 \gg \mathrm{S}, 72^{\circ} 36>47 \gg 0\right)$, PN Bernardo O'Higgins (incluyendo los valles Bernardo y Huemules)

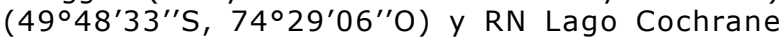

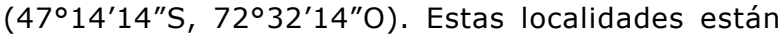
caracterizadas por bosques de Nothofagus pumilio y Nothofagus dombeyi y estepa patagónica. Las muestras de la región de Magallanes fueron obtenidas del PN Torres del Paine ( $\left.50^{\circ} 57^{\prime} 38^{\prime \prime} \mathrm{S}, 73^{\circ} 24^{\prime} 51^{\prime \prime} \mathrm{O}\right)$ y el valle Katraska, ubicado en el PN Bernardo O'Higgins (48 $42^{\prime} 54^{\prime \prime} S$, $\left.74^{\circ} 00^{\prime} 37^{\prime \prime O}\right)$. Esta zona posee un clima templado frío con bosques siempre verdes y turberas.

Análisis de muestras. Todas las muestras colectadas $(n=200)$ fueron analizadas en busca de huevos y ooquistes de parásitos mediante las técnicas de sedimentación y flotación simple.

La flotación simple consistió en mezclar $10 \mathrm{~g}$ de muestra con $10 \mathrm{~mL}$ de formalina al $10 \%$. La mezcla se dejó reposar por 30 minutos, luego se filtró a través de una capa de gaza y se introdujo en un tubo cónico de vidrio de $15 \mathrm{~mL}$, el cual fue llenado casi por completo con solución salina. La mezcla se centrifugó por 10 minutos a 1.500 rpm y el sobrenadante fue eliminado. El tubo se llenó nuevamente con solución salina y se repitió el proceso de centrifugado una vez más para lavar la muestra y obtener un sedimento de aproximadamente $1 \mathrm{~mL}$. El sedimento fue resuspendido con $12 \mathrm{~mL}$ de solución de sulfato de zinc y centrifugado por 2 minutos a $1.500 \mathrm{rpm}$. El tubo fue posicionado verticalmente en una gradilla y se llenó con sulfato de zinc formando un menisco invertido. Finalmente, un cubreobjetos se posicionó sobre el menisco por 10 minutos para captar formas parásitas.

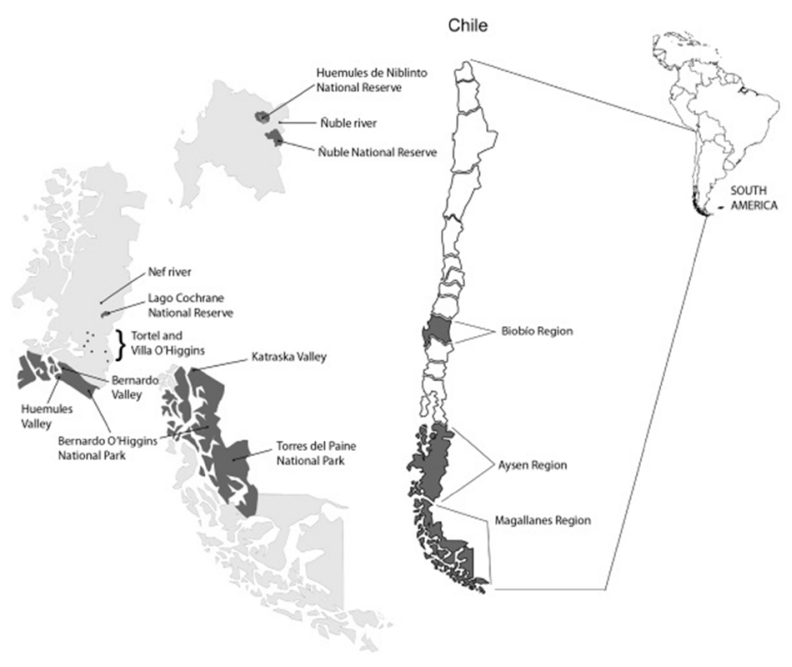

Figura 1. Mapa de Chile con las localidades donde las muestras de heces de huemul (Hippocamelus bisulcus) fueron recolectadas. 
En la técnica de sedimentación, se repitió el mismo procedimiento utilizado en la flotación simple hasta el primer centrifugado. Posteriormente, el sedimento obtenido se resuspendió en $7 \mathrm{~mL}$ de formalina al $10 \%$. Luego, se adicionó $4 \mathrm{~mL}$ de etil-acetato, se agitó la mezcla por 30 segundos y se centrifugó por 10 minutos a 1.500 $\mathrm{rpm}$. El sobrenadante fue removido y se depositó dos gotas de sedimento en portaobjetos separados, donde uno fue teñido con Lugol y el otro se dejó sin teñir (6). Adicionalmente, se evaluaron 124 muestras para Fasciola hepatica utilizando la técnica de sedimentación previamente descrita y la adición de 3 gotas de tinción verde de malaquita (1\%) al sedimento (7).

Análisis estadístico. Se calculó la prevalencia de parásitos, definida como el porcentaje de muestras de heces con al menos una especie de parásito presente, en relación al total de muestras analizadas. Se compararon los valores absolutos de prevalencia de cada estación mediante el estadístico paramétrico chi-cuadrado con corrección de Yates y se consideró un nivel de significación de $p \leq 0,05$ y un $95 \%$ de confiabilidad.

Aspectos éticos. La investigación fue autorizada por el Servicio Agrícola y Ganadero de Chile (SAG) y aprobada por el Comité de Bioética de la Facultad de Ciencias Veterinarias de la Universidad de Concepción.

\section{RESULTADOS}

De las 200 muestras analizadas, el 44\% (88/200) resultó positiva a alguna forma parasitaria. Entre éstas, se identificaron cuatro tipos de parásitos, registrándose huevos tipo Estrongílido, huevos de Nematodirus sp. (Nematoda), huevos de Moniezia sp. (Cestoda)(Figura 2) y ooquistes de Eimeria spp. Se observaron infecciones mixtas de huevos tipo Estrongílido y de Nematodirus sp. en 4 muestras de Aysén y 6 muestras de Magallanes, mientras que infecciones con huevos tipo Estrongílido, Nematodirus sp. y Moniezia sp. fueron evidenciadas en sólo 2 muestras de Aysén (Figura 3). No se observó la presencia de $F$. hepatica en las muestras analizadas. El detalle sobre la prevalencia de los distintos parásitos en las heces se muestra en la Tabla 1.

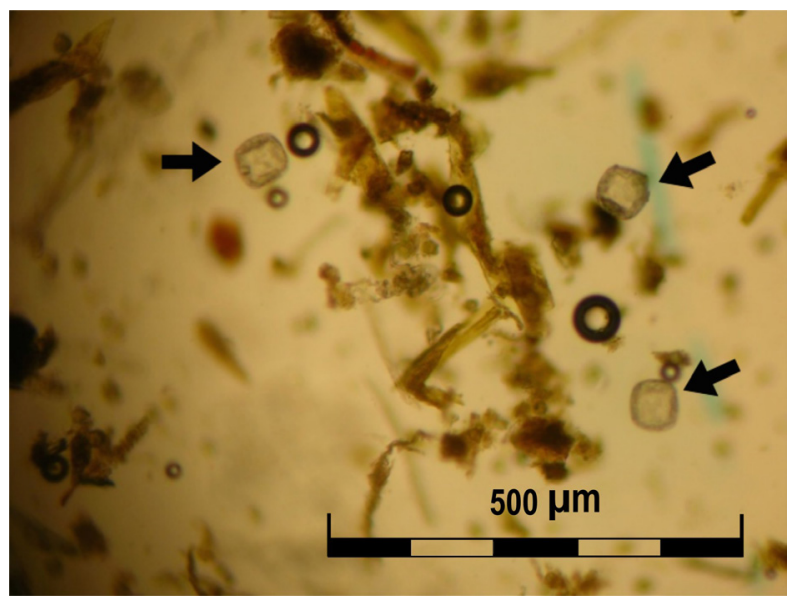

Figura 2. Se observa la presencia de huevos de Moniezia sp. (flechas) en heces de huemul (Hippocamelus bisulcus).
Tabla 1. Prevalencia de huevos de parásito y ooquistes en muestras de heces de huemul (Hippocamelus bisulcus) en distintas regiones de Chile.

\begin{tabular}{ccccc}
\hline \multirow{2}{*}{ Parásitos } & \multicolumn{4}{c}{ Región } \\
\cline { 2 - 5 } & $\begin{array}{c}\tilde{\text { Nuble }}(\mathbf{n = 1 7 )} \\
\text { Tipo Estrongílido }\end{array}$ & $\begin{array}{c}\text { Aysén } \\
(\mathbf{n = 1 2 3})\end{array}$ & $\begin{array}{c}\text { Magallanes } \\
(\mathbf{n = 6 0 )}\end{array}$ & $\begin{array}{c}\text { Total } \\
(\mathbf{n = 2 0 0})\end{array}$ \\
Nematodirus sp. & 0 & $20.3(25)$ & $73.3(44)$ & $34.5(69)$ \\
Moniezia sp. & $11.8(2)$ & 0 & $3.3(2)$ & $2.5(5)$ \\
Total & $11.8(2)$ & $22.8(28)$ & $76.7(46)$ & $38.0(76)$ \\
\hline
\end{tabular}

Número de muestras positivas ( ).

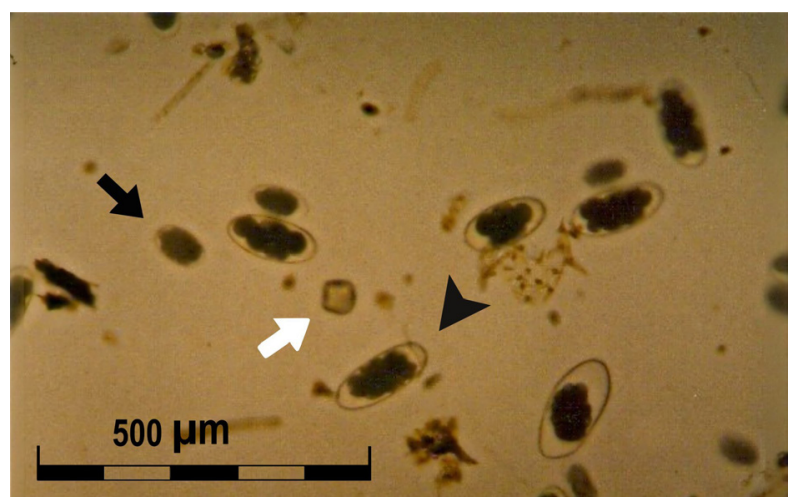

Figura 3. Se observa la presencia de huevos tipo Estrongílido (flecha negra), Moniezia sp. (flecha blanca) y huevos de Nematodirus sp. (cabeza de flecha) en muestras de heces de huemul (Hippocamelus bisulcus).

Respecto a la estacionalidad en la presentación de infecciones parasitarias, se detectaron formas parásitas en todas las estaciones del año, con mayores prevalencias durante otoño y primavera (Tabla 2). Se determinaron infecciones mixtas de huevos tipo Estrongílido y de Nematodirus sp. en 6 muestras obtenidas en otoño y 4 muestras en primavera, mientras que infecciones con huevos de Nematodirus sp., de tipo Estrongílido y Moniezia sp. fueron observadas en sólo 2 muestras de primavera. El análisis de prevalencia parasitaria entre estaciones del año para las regiones de Aysén y Magallanes, demostró diferencias significativas entre todas las estaciones $(p \leq 0.05)$.

Tabla 2. Prevalencia de huevos de parásito y ooquistes en muestras de heces de huemul (Hippocamelus bisulcus) entre las distintas estaciones del año.

\begin{tabular}{lccccc}
\hline \multirow{1}{*}{ Parásitos } & \multicolumn{5}{c}{ Estación } \\
\cline { 2 - 6 } & $\begin{array}{c}\text { Otoño } \\
(\mathbf{n = 4 0 )}\end{array}$ & $\begin{array}{c}\text { Invierno Primavera } \\
(\mathbf{n = 6})\end{array}$ & $\begin{array}{c}\text { Verano } \\
(\mathbf{n = 1 9 )}\end{array}$ & $\begin{array}{c}\text { Total } \\
(\mathbf{n = 1 1})\end{array}$ \\
\hline Tipo Estrongílido & $95.0(38)$ & $100(6)$ & $84.2(16)$ & $81.8(9)$ & $90.8(69)$ \\
Nematodirus sp. & $2.5(1)$ & 0 & $15.8(3)$ & $9.1(1)$ & $6.6(5)$ \\
Moniezia sp. & $2.5(1)$ & 0 & 0 & $9.1(1)$ & $2.6(2)$ \\
\hline
\end{tabular}

Número de muestras positivas ( ). 


\section{DISCUSIÓN}

Se observó una mayor prevalencia y diversidad de parásitos en las muestras de Aysén y Magallanes. Lo anterior, puede deberse al clima más húmedo $\mathrm{y} / \mathrm{o} a$ la mayor densidad de huemules presentes en la zona $(1,2)$, lo que puede favorecer las interacciones con ungulados domésticos y silvestres y, en consecuencia, la diseminación de parásitos al huemul. En ambas regiones, se detectó también la presencia de Nematodirus sp., el cual es un endoparásito presente comúnmente en el ganado ovino. Infecciones severas con Nematodirus sp. pueden resultar en pérdida de peso, deshidratación, edema submandibular y muerte en corderos (8), sin embargo, hasta la fecha no existen reportes que indiquen consecuencias patológicas de este parásito en el huemul. Los huevos tipo Estrongílido fueron altamente prevalentes y se identificaron en todas las estaciones (Tabla 2), lo que podría estar relacionado con la gran capacidad de estos huevos de resistir bajas temperaturas y humedad (9).

La ausencia de huevos en el $56 \%$ de las muestras podría ser consecuencia de un período de transporte prolongado, lo que llevaría a subestimar formas parasitarias menos resistentes al ambiente. La técnica utilizada es también una limitante de este estudio, ya que permite identificar formas parasitaras sólo hasta nivel de género, por lo que se recomienda que futuras investigaciones utilicen técnicas que permitan el reconocimiento de especies.

La baja detección y diversidad de parásitos en la región de Ñuble, puede ser consecuencia de la reducida carga animal en el ambiente, ya que la zona muestreada fue por varios años "zona de exclusión de ganado". También debe considerarse la baja densidad de huemules en esta zona, en la cual se ha descrito una población extremadamente reducida, lo que podría suponer una menor diseminación de parásitos entre individuos, disminuyendo así las probabilidades de infección $(1,2)$. En la región de Ñuble, sólo fue registrada la presencia de huevos de Moniezia sp., género descrito previamente mediante análisis coprológicos de huemules presentes en esta zona (5). Los huevos de Moniezia sp. se encontraron sólo en otoño y verano. Estudios más acabados debieran comprobar si la combinación entre precipitación y alza de las temperaturas de estas estaciones inciden en la mayor contaminación de las praderas o aumento de la densidad de ácaros oribátidos (hospedadores intermediarios de Moniezia) en estas localidades, lo que podría influenciar la propagación de Moniezia sp. en la zona. Por otro lado, es importante considerar que la baja diversidad de parásitos encontrados durante este estudio en la región de Ñuble puede corresponder a un sesgo del estudio, dado el reducido número de muestras analizadas, por lo que no se puede descartar la presencia de parásitos descritos previamente para la zona, como son coccidias y huevos tipo Estrongílido (3).

Fasciola hepatica no fue detectada durante esta investigación, pero sí ha sido reportada previamente en huemules mantenidos en cautiverio (4). En Chile, la fasciolasis es altamente prevalente entre las regiones de Coquimbo y La Araucanía, lo que contrasta con el escenario presente al sur del país, particularmente en Magallanes, donde el agente causal aún no ha sido reportado (10). La ausencia de este parásito en la región de Ñuble puede ser consecuencia de la ausencia de su hospedador intermediario en las zonas incluidas en este estudio, hecho que debe ser investigado.

En conclusión, el presente estudio entrega información acerca de los parásitos presentes en las poblaciones de huemul distribuidas en el centro y sur de Chile. Esta información es relevante para la conservación del huemul debido a las perturbaciones antropogénicas que actualmente afectan a ambas poblaciones. No existen aún estudios que evalúen los efectos patológicos que puedan tener Moniezia spp., Eimeria spp. y Nematodirus $\mathrm{sp}$. en los huemules, lo cual resulta de gran relevancia en el contexto actual de competencia constante por recursos con el ganado y otros factores antropogénicos, particularmente para las poblaciones de huemul presentes en Ñuble. Cabe destacar que es necesario continuar con monitoreos parasitológicos en poblaciones de huemul, sobre todo en aquellas que comparten hábitat con especies domésticas, para así comprender mejor los mecanismos de transmisión de agentes infecciosos y parasitarios entre el ganado y el huemul.

\section{REFERENCIAS}

1. Vila AR, Saucedo C, Aldridge D, Ramilo E, Corti P. South Andean huemul Hippocamelus bisulcus (Molina 1782). In: Duarte JM, González S. Neotropical Cervidology. IUCN: Jaboticabal, Brazil; 2010. https://www.iucn.org/es/content/ neotropical-cervidology

2. Corti $P$, Shafer AB, Coltman DW, Festa-Bianchet $M$. Past bottlenecks and current population fragmentation of endangered huemul deer (Hippocamelus bisulcus): implications for preservation of genetic diversity. Conserv Genet. 2011; 12(1):119-128. https://doi.org/10.1007/ s10592-009-9997-7
3. Texera WA. Algunos aspectos de la biología del huemul en cautividad. Ans Inst Pat. 1974; 5(1-2):155-188. http://www.bibliotecadigital.umag.cl/bitstream/ handle/20.500.11893/567/Texera Anales 1974 vol5 p.155-188.pdf?sequence $=1$ \&isAllowed $=y$

4. Fugassa MH. Checklist of helminths found in Patagonian wild mammals. Zootaxa. 2015; 4012(2):271-328. https://doi.org/10.11646/ zootaxa.4012.2.3 
5. Beltrame MO, Tietze $E$, Pérez $A E$, Bellusci $A$, Sardella NH. Ancient parasites from endemic deer from "CUEVA PARQUE DIANA" archeological site, Patagonia, Argentina. Parasitol Res. 2017; 116(5):1523-1531. https://doi.org/10.1007/ $\underline{\text { s00436-017-5429-7 }}$

6. Leventhal R, Cheadle RF. Medical parasitology: a self-instructional text. FA Davis: Philadelphia; 2012.

7. Flanagan AM, Edgar HWJ, Forster F, Gordon A, Hanna REB, McCoy $M$ et al. Standardisation of a coproantigen reduction test (CRT) protocol for the diagnosis of resistance to triclabendazole in Fasciola hepatica. Vet Parasitol. 2011; 176(1):3442. https://doi.org/10.1016/j.vetpar.2010.10.037
8. Caballero AJA, Sarmiento RC, Acosta JFT, Castro CS. El control de los nemátodos gastrointestinales en caprinos: ¿dónde estamos? Bioagrociencias. 2011; 4(2):1-16. http://www.ccba.uady. mx/bioagro/ v4N2/archivo\%202.pdf

9. Turner WC, Getz WM. Seasonal and demographic factors influencing gastrointestinal parasitism in ungulates of Etosha National Park. J Wildl Dis. 2010; 46(4):1108-1119. https://doi.org/10.7589/0090$\underline{3558-46.4 .1108}$

10. Valenzuela G, Quintana I. Evolución de huevos de Fasciola hepatica en el medio ambiente en Temuco, IX Región de Chile. Arch Med Vet. 1998; 30(1):109-114. https://doi.org/10.4067/S0301732X1998000100012 